\title{
SEROPREVALENCE OF IgG TOXOPLASMA IN HIV INFECTED PATIENTS
}

\author{
Sheela Mathew ${ }^{1}$, Shamlath ${ }^{2}$
}

1Additional Professor, Department of Infectious Diseases, Government Medical College Hospital, Kozhikode, Kerala. 2MSc Microbiology Student, Department of Medical Microbiology, School of Health Sciences, University of Calicut, Kerala.

\section{ABSTRACT}

\section{BACKGROUND}

Toxoplasmosis is a zoonotic infection in humans caused by an intracellular parasite Toxoplasma gondii. It is a highly prevalent infection, particularly in immune suppressed people. Infection with Toxoplasma in immune-competent people is generally without symptoms. Infection can be severe in immune compromised individuals with AIDS, in whom infection can lead to cerebral toxoplasmosis. Patients with CD4 counts of less than 100 cells/ $\mu \mathrm{L}$ and T. gondii IgG positivity have a $30 \%$ risk of reactivation of disease, if prophylaxis is not given or immune function is not restored.

The aim of the study was to find out the seroprevalence of Toxoplasma IgG antibodies among HIV infected persons in the community.

\section{MATERIALS AND METHODS}

This was an observational study. Serological level of Toxoplasma IgG antibodies using ELISA technique in 30 HIV positive patients was done. IgG antibodies were examined with Euro Immune anti-T. gondii ELISA kit, Positive titre as per manufacturer's instruction was $>11 \mathrm{IU} / \mathrm{mL}$. HIV testing was done in VCTC centres and the group included 21 males and 9 females.

\section{RESULTS}

The age varied from 20 - 60 and none of them had any neurological involvement suggestive of toxoplasmosis. All patients were on HAART. Nobody had a previous IgG Toxoplasma estimation. The CD4 count varied from $4-929$, but 17 of 30 had CD4<200 who were receiving cotrimoxazole prophylaxis. 12/30 showed a positive titre. This included 8 males and 4 females. $75 \%$ were below 40 yrs. Maximum titre noted was $250.4 \mathrm{IU} / \mathrm{mL} .8 / 12$ had titre $>100$.

\section{CONCLUSION}

$40 \%$ of HIV positive patients showed a positive serology. Toxoplasmosis being commonest opportunistic infection among HIV patients who develop reactivation of infection when CD4 falls below 200, it is ideal that all patients with HIV infection have a baseline estimation of antibody titre. This titre should be rechecked when CD4 falls below 200, because prompt identification and management helps to decrease neurological sequelae and early recovery. Those who have a negative test should be advised preventive behavioural practices. Small sample size selected was the limitation of the study. Hence, further studies including a better sample size, selecting from particular geographical areas and comparing with lifestyles such as socioeconomic status, environmental factors, cooking habits etc. should be done to assess the prevalence and risk factors in different geographical areas.

\section{KEYWORDS}

Toxoplasmosis, Toxoplasma IgG, Seroprevalence, HIV Infection. HOW TO CITE THIS ARTICLE: Mathew S, Shamlath. Seroprevalence of IgG toxoplasma in HIV infected patients. J. Evolution Med. Dent. Sci. 2018;7(09):1148-1151, DOI: 10.14260/jemds/2018/261

\section{BACKGROUND}

Toxoplasmosis is a zoonotic infection in humans caused by an intracellular parasite Toxoplasma gondii, a sporozoan protozoa belonging to genus of Toxoplasma. Cats are the primary hosts, while humans and other mammals serve as intermediate hosts. Infection with T. Gondii is common among humans and it is estimated that one-third of the world's population has been exposed. The seroprevalence varies widely in different regions and depends on socio-economic status, environmental factors and meat-cooking habits. ${ }^{1}$ Indian studies of prevalence of toxoplasmosis reveal a wide variation and one study reported prevalence as high as $77 \%$ in women of reproductive age group. The average prevalence among Indian pregnant women is $7.7 \%$.

'Financial or Other Competing Interest': None.

Submission 16-01-2018, Peer Review 14-02-2018,

Acceptance 20-02-2018, Published 26-02-2018.

Corresponding Author:

Dr. Sheela Mathew,

Government Medical College Hospital,

Kozhikode, Kerala.

E-mail: shaarongardens@yahoo.com

DOI: $10.14260 /$ jemds $/ 2018 / 261$
There is no published data on the disease prevalence in Kerala. A pilot study done in Kozhikode district revealed a seroprevalence of $27.16 \%$ (unpublished data) in immune competent persons. The protozoan was first discovered by Nicolle and Manceaux in 1908. They first isolated it from African rodents Ctenodactylus gundi. It was identified as an agent of infectious disease in 1932. In 1983 toxoplasmosis was more widely recorded as a cause of morbidity in immune deficient patients including AIDS and idiopathic CD4 cytopenia syndrome. ${ }^{2}$ Most infections are however subclinical and disease typically becomes apparent only as a congenitally acquired infection and in patients with significant immunodeficiency such as in acquired immunodeficiency syndrome (AIDS). 3,4

\section{Toxoplasma gondii occurs in 3 Stages ${ }^{5}$}

1. Tachyzoites, also called trophozoite is the rapidly multiplying form and can infect any cell in the body.

2. Bradyzoites and tissue cyst is a stage encysted in the tissue.

3. Oocyst is a cyst surrounded by a thick resistant wall. It is seen in definitive hosts formed by sexual reproduction. 
Trophozoite and tissue cyst represent stages in asexual reproduction.

Humans acquire infection via eating undercooked or raw meat infected with tissue cysts, via ingestion of food or water contaminated with infected cat faeces carrying sporulated oocysts. ${ }^{1}$ Maternal to foetus transmission can also occur. ${ }^{4}$ Rare cases of individuals becoming infected through blood transfusions or organ transplantation have also been reported. Sporozoites from oocysts and bradyzoites from tissue cysts invade intestinal mucosa and multiply as tachyzoites in epithelial cells. Tachyzoites spread to mesenteric lymph nodes and then via blood stream and lymphatics reach organs such as brain, eye, liver, spleen, heart, skeletal muscle, lymph nodes and placenta of pregnant mother. Focal areas of necrosis develop in these organs. 5 With the development of immunity, tachyzoites are destroyed and acute infection resolves. Some of the tachyzoites may still persist and develop into tissue cysts containing bradyzoites, which remain viable for years. When there is suppression of immune system, infection is reactivated.

Development of both humoral and cellular immunity alters the course of toxoplasma infection and its clinical manifestations. Humoral immunity is characterised by the production of specific circulating antibodies, both IgM and IgG. Toxoplasma specific IgM antibodies appear first, hence its presence is suggestive of an acute infection. IgG antibodies appear late and persist, and is suggestive of a chronic infection. Cell mediated immunity through activated macrophages and monocytes is suggested to play an important role in conferring resistance to re-infection. This stage is associated with the disappearance of tachyzoites from various tissues, especially from extraneural tissues and formation of tissue cysts. The tachyzoites may persist in the central nervous system and in the eye due to the absence of circulating antibodies in the tissues. 6

Infection in the immune-competent host - In humans, asymptomatic or benign toxoplasmosis is the rule in immune competent patients. ${ }^{3}$ Eighty to ninety percent of T. gondii infections in immune competent hosts are asymptomatic. Generally, when acute infection is symptomatic, manifestations include fever, nonspecific rash and lymphadenopathy. The most common presentation of symptomatic postnatally acquired toxoplasmosis in immune competent patients is painless cervical adenopathy. When fever and lymph node enlargement persists, often it ends in biopsy studies and the common histological picture is necrosis with microgranulomas. In majority of cases, the clinical course is benign and symptoms and signs resolve within a few weeks. However, severe manifestations of infection including chorioretinitis can occur in some immunecompetent hosts. ${ }^{3}$

Chorioretinitis or ocular toxoplasmosis is a relatively common manifestation of $\mathrm{T}$. gondii infection. ${ }^{6}$ Ocular toxoplasmosis occurs when cysts deposited in or near the retina become active, producing tachyzoites. Focal necrotising retinitis is the characteristic lesion, but retinal scars from prior reactivation are typically present. Clinical presentation usually is eye pain and decreased visual acuity. Adults who had acquired disease in infancy usually present with bilateral eye involvement. Adults with acute infection at a later age generally present with unilateral ocular involvement. Depending on the location and severity of toxoplasma chorioretinitis, infection can result in permanent retinal scarring and loss of visual acuity. Recurrent episodes are common, resulting in multiple areas of retinal scarring and functional loss.

Studies have shown that in high endemic areas, there is an association between a typical genotype and severe toxoplasmosis acquired by immune competent adults. ${ }^{7}$ It has also been recently reported that an unusual abundance of atypical strains in the environment is associated with human ocular toxoplasmosis and with severe forms of congenital toxoplasmosis. ${ }^{7}$

Congenital infection- Approximately 10\% - 20\% of pregnant women infected with $\mathrm{T}$. gondii have clinical symptoms of the disease. ${ }^{8}$ The most common signs of infection are lymphadenopathy and fever. If the mother was infected prior to pregnancy, there is virtually no risk of foetal infection as long as she remains immune competent. If the infection is acquired during the pregnancy, there is risk of infection to the foetus. The rate of transplacental infection has been estimated to be $50 \%$ for untreated mothers and $25 \%$ for treated mothers.

The rate of foetal infection varies with trimester with $10 \%-25 \%$ of infections occurring in the first trimester, $30 \%$ in the second trimester and $50 \%$ in the third trimester. ${ }^{8}$ Infection during the first or second trimesters appears to be most severe. The clinical features of congenitally acquired T.gondii infection include chorioretinitis, blindness, seizures, microcephaly, anaemia and encephalitis. Infections acquired during the third trimester are usually subclinical; however, clinical disease may still occur later in life. $75 \%$ of infants congenitally infected with $\mathrm{T}$. gondii manifest no symptoms, $14 \%$ had evidence of chorioretinitis and 9\% demonstrate signs of CNS involvement.

Infections in immune compromised patients- Most cases of toxoplasmosis in immune compromised patients are a consequence of latent infection and reactivation. ${ }^{9}$ In patients with AIDS, T. gondii tissue cysts can reactivate with CD4 counts less than 200 cells $/ \mu \mathrm{L}$. When CD4 counts are less than 100 cells $/ \mu \mathrm{L}$, clinical disease is more likely. Patients with CD 4 counts less than 100 cells $/ \mu \mathrm{L}$ and $\mathrm{T}$. gondii IgG positivity have a $30 \%$ risk of eventually developing reactivation disease if adequate prophylaxis is not given or immune function is not restored. Although, toxoplasmosis in immune compromised patients may manifest as chorioretinitis, reactivation disease in these individuals is typically in the central nervous system with brain involvement being common. ${ }^{9}$

Toxoplasma encephalitis and brain abscess presents most commonly as headache, focal neurologic deficits and seizures. ${ }^{2}$ Lumbar puncture studies performed when there is no evidence of raised ICT, reveals a picture mimicking tuberculous meningitis. With significant disease, patients may also have the signs and symptoms of elevated intracranial pressure. Cerebral toxoplasmosis is generally identified on CECT scan as multiple rim enhancing lesions; solitary lesions may also be seen. Absence of lesions in CT or MRI scans does not rule out the diagnosis of central nervous system toxoplasmosis. When plain CT scan of a patient reveals an infarct like picture contrast study must be done, as many cases are misdiagnosed as vascular events. In a patient who is immune suppressed and has focal neurologic deficits, if CT scan is normal MRI scan is advisable. ${ }^{9}$ 
Apart from central nervous system toxoplasmosis, toxoplasma pneumonia, myocarditis as well as disseminated toxoplasmosis are also commonly identified in immune compromised patients. Toxoplasma pneumonia typically presents with symptoms, which are typical for an infectious pulmonary process. Symptoms include fever, dyspnoea and cough. Chest radiography is often non-specific, but findings may be similar to that of Pneumocystis jiroveci pneumonia. Diagnosis is established via bronchoalveolar lavage (BAL). Most patients with extra-central nervous system manifestations of toxoplasmosis will also be noted to have central nervous system lesions when appropriate radiographic studies have been performed. Toxoplasma encephalitis and brain abscess can result in permanent neurologic sequelae depending on the location of the lesion and the extent of local damage and inflammation. ${ }^{2}$

Diagnosis is confirmed by isolation of toxoplasma from blood or body fluids, demonstration of parasite in tissues, detection of specific nucleic acid sequence with DNA probes or detection of toxoplasma specific immunoglobulin.10 Polymerase chain reaction (PCR) amplification is used to detect the DNA in body fluids and tissues. Amniotic fluid, brain tissue, BAL fluid, cerebrospinal fluid, vitreous and aqueous fluid, urine and peripheral blood can be used for PCR. IgM levels are estimated to demonstrate acute infection. IgG levels are assayed for diagnosing past infection. Many tests for avidity of Toxoplasma IgG antibodies have been introduced to differentiate between recently acquired and distant infection. ${ }^{10}$

\section{Aim of the Study}

To find out the seroprevalence of Toxoplasma IgG antibodies among HIV infected persons attending the ART clinic of our Institution, Govt. Medical College Hospital, Kozhikode, Kerala that is catering to six northern districts of Kerala.

\section{MATERIALS AND METHODS}

This was an observational study conducted in $30 \mathrm{HIV}$ positive patients who were attending ART Clinic of Govt. Medical College Hospital, Kozhikode, Kerala. Patients were given an awareness session in one of the group meetings of positive people. In the awareness session, the chances of getting various opportunistic infections in HIV/ AIDS including Toxoplasmosis was discussed and the methods of detection was also informed. In a subsequent session, the willingness of testing for Toxoplasmosis was discussed and samples were collected from those people who expressed willingness for testing.

Serological levels of Toxoplasma IgG antibodies using ELISA technique in $30 \mathrm{HIV}$ positive patients were done. IgG antibodies were examined with Euro Immune anti-T. gondii ELISA kit. Antibody levels were evaluated by following the instructions of manufacturers. Results were expressed in titres. Control sera and calibration sera were not diluted. Results were read by photometric measurements of the colour intensity at a wave length of $450 \mathrm{~nm}$ and reference wave length between 620 and $650 \mathrm{~nm}$ within 30 minutes of adding stop solution. Positive titre as per manufacturer's instruction was $>11 \mathrm{IU} / \mathrm{mL}$. HIV testing was done in VCTC centres and the group included 21 males and 9 females.
Testing was on a voluntary basis; hence, sample did not include any from positive children.

\section{RESULTS}

The study population included 21 males $(70 \%)$ and 9 females (30\%). Age varied from 20 - 60. Out of 30 patients, 21 belonged to $<40$ age group (70\%) and 9 belonged to $41-60$ years (30\%).

None of them had any neurological involvement suggestive of toxoplasmosis. All patients were on HAART. Nobody had a previous IgG Toxoplasma estimation.

The CD4 count varied from 4 - 929. This included the last available CD4. 17 patients $(56.67 \%)$ had CD4 $<200$ who were receiving cotrimoxazole prophylaxis. 10 (33.33\%) had CD4 between 200 - 499. 3 (10\%) had CD4 > 500. The last 2 groups had $2^{\text {nd }}$ or $3^{\text {rd }} \mathrm{CD} 4$ count, which was more than 200 and their baseline was $<200$.

$12 / 30(40 \%)$ showed a positive titre. This included 8 males and 4 females. Out of 21 males 8 were positive (43\%), whereas out of 9 females 4 were positive (44\%). Hence, there was no significant gender variation. $75 \%$ were below 40 yrs. Maximum titre noted was $250.4 \mathrm{IU} / \mathrm{mL}$. 8/12 had titre $>100$.

\begin{tabular}{|c|c|c|}
\hline Result & Number & Percentage \\
\hline Positive & 12 & 40 \\
\hline Negative & 18 & 60 \\
\hline Total & $\mathbf{3 0}$ & $\mathbf{1 0 0}$ \\
\hline Table 1. Prevalence of Anti-Toxoplasma Antibody \\
among Test Community \\
\hline
\end{tabular}

\section{Prevalence of anti Toxoplasma antibody among test community}

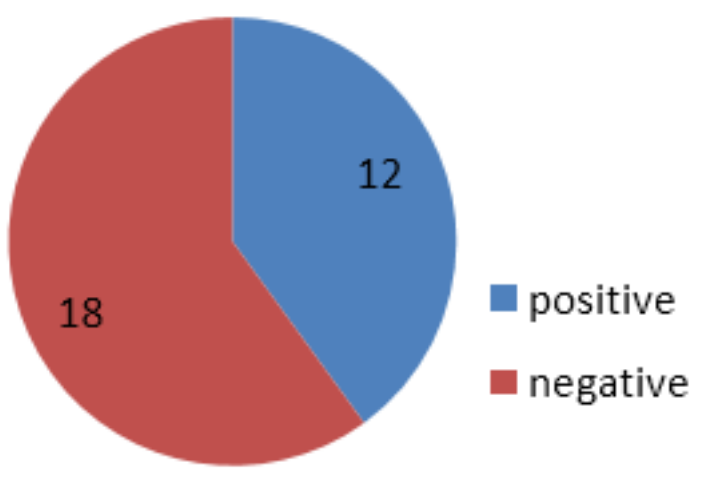

\begin{tabular}{|c|c|c|c|}
\hline Gender & Total Tested & Positive & Prevalence \\
\hline Male & 21 & 8 & $43 \%$ \\
\hline Female & 9 & 4 & $44 \%$ \\
\hline Table 2. Gender Wise prevalence of Toxoplasma Antibody
\end{tabular}

Table 2. Gender Wise prevalence of Toxoplasma Antibody

\begin{tabular}{|c|c|r|}
\hline Age & & \% \\
\hline $20-40$ & 9 & 75 \\
\hline $41-60$ & 3 & 25 \\
\hline \multicolumn{2}{|c|}{ Table 3. Age distribution of Positive Cases } \\
\hline
\end{tabular}

\section{DISCUSSION}

$40 \%$ of HIV positive patients showed a positive serology. The seroprevalence varies widely in different regions and depends on socio-economic status, environmental factors and 
meat-cooking habits. Since the prevalence varies widely from region to region, we should have a prevalence rate in the community as a whole for a comparison between immune competent and compromised patients. There was no comparable study available showing seroprevalence in immune competent group in this community.

There was no significant male-female variation in prevalence. This shows both sexes are having equal risk exposure and equally affected by the disease. However, with a small sample size it is difficult to conclude the equal prevalence. Selecting equal number is ideal. Since both sexes are having equal prevalence, both genders should be counselled to minimise risk behaviour.

$75 \%$ of positive patients were below age 40 . This shows seroconversion occurs at a younger age group in our community. This was in contrast to other studies, which showed increase in titres as age advanced. There is a close relationship between the incidence of toxoplasmosis and the seroprevalence of toxoplasma antibodies within a population. All ages can be affected; hence, evidence of infection becomes more as age advances.

Toxoplasmosis being commonest opportunistic infection among HIV patients who develop reactivation of infection when CD4 falls below 200. It is ideal that all patients with HIV infection have a baseline estimation of antibody titre. This titre should be rechecked when CD4 falls below 200, because prompt identification and management helps to decrease neurological sequelae and early recovery.

Those who have a negative test should be advised preventive behavioural practice such as wearing gloves while gardening or handling soil. Cats often use gardens and sand boxes as litter boxes and they can pass the parasite in the faeces. Hands should be washed with soap and water after handling raw meat, after handling soil and before preparing and eating food. Pregnant females may avoid handling raw meat, the cutting boards and utensils used to cut meat should be washed promptly. Meat should be cooked until it is no longer pink in the centre or until juice run clear. Never sample meat before it is fully cooked. Avoid handling stray cats or kittens.

\section{CONCLUSION}

Since neuroimaging, PCR, organ biopsy and histopathology all are costly and time consuming and 97.5\% - 100\% of HIV infected patients with central nervous system toxoplasmosis have positive serology. This simple test forms an important one in diagnosing central nervous system toxoplasmosis. Diagnosis and management may be done in a compatible clinical setting with positive serology, and if possible neuroimaging. A rise in IgG value from baseline will give a clue in diagnosis. Since $40 \%$ of HIV positive patients show IgG positivity, based on a single test diagnosis cannot be established.

Sample size selected was small because of lack of willingness of patients. Hence, further studies including a better sample size, selecting from particular geographical areas and comparing with lifestyles such as socio-economic status, environmental factors, cooking habits etc. should be done to assess the prevalence and risk factors in different geographical areas.

\section{REFERENCES}

[1] Ortega Y. Toxoplasmosis. In: Ortega YR. edr. Foodborne parasites. New York: Springer 2006.

[2] Israelski DM, Remington JS. Toxoplasmic encephalitis in patients with AIDS. Infect Dis Clin North Am 1988;2(2):429-45.

[3] Carme B, Bissuel F, Ajzenberg D, et al. Severe acquired toxoplasmosis in immunocompetent adult patients in French Guiana. J Clin Microbiol 2002;40(11):4037-44.

[4] Jones J, Lopez A, Wilson M. Congenital toxoplasmosis. Am Fam Physician 2003;67(10):2131-8.

[5] Dubey JP. Advances in the life cycle of Toxoplasma gondii. International Journal for Parasitology 1998;28(7):1019-24.

[6] Grigg ME, Ganatra J, Boothroyd JC, et al. Unusual abundance of atypical strains associated with human ocular toxoplasmosis. J Infect Dis 2001;184(5):633-9.

[7] Ajzenberg D, Cogne N, Paris L, et al. Genotype of 86 Toxoplasma gondii isolates associated with human congenital toxoplasmosis and correlation with clinical findings. J Infect Dis 2002;186(5):684-9.

[8] Chakraborty P, Sinha S, Adhya S, et al. Toxoplasmosis in women of child bearing age and infant follow up after in-utero treatment. Indian J Pediatr 1997;64(6):879-82.

[9] Takashima Y, Suzuki K, Xuan X, et al. Detection of the initial site of Toxoplasma gondii reactivation in brain tissue. Int J Parasitol 2008;38(5):601-7.

[10] Remington JS, Thulliez P, Montoya JG. Recent developments for diagnosis of toxoplasmosis. J Clin Microbiol 2004;42(3):941-5. 